\title{
Wheel Obstraction Detection with Machine Learning
}

\author{
Shanmukhsrivamsinimmagadla, P.Shanmuga Prabha
}

\begin{abstract}
In this paper, to blessing an ongoing programmed innovative and insightful based absolutely rail assessment framework, which plays examinations at sixteen $\mathrm{km} / \mathrm{h}$ with a casing rate of 20 fps. The framework identifies significant rail segments including ties, tie plates, and grapples, with high exactness and productivity. To accomplish this objective, to initially widen an immovable of picture and video investigation and after that prompt a particular worldwide streamlining structure to join proof from two or three cameras, Global Positioning System, and separation size apparatus to moreover improve the recognition execution. Additionally, as the grapple is a significant kind of rail clasp, to've as needs be propelled the push to hit upon stay special cases, which consolidates evaluating the grapple circumstances on the tie stage and recognizing grapple design exemptions on the consistence level. Quantitative examination performed on a huge video certainties set caught with unmistakable tune and lighting installations conditions, notwithstanding on a continuous order check, has affirmed empotoring execution on each rail perspective recognition and stay special case location. In particular, a middle of $94.67 \%$ accuracy and ninety three\% remember expense has been finished for recognizing each of the 3 rail segments, and a 100\% recognition charge is practiced for consistence level stay special case with three phony positives predictable with hour. To our excellent comprehension, our framework is the essential to address and clear up both perspective and special case location issues in this rail assessment region.
\end{abstract}

Index Terms-Anchor exception detection, system imaginative and prescient generation, multisensory proof integration.

\section{INTRODUCTION}

To maintain railroads need to investigate their tracks for bodily defects on a regular basis. Additionally enforced by means of character railroad businesses, normally with extra stringent requirements, to preserve song health to a higher standard. Such a track inspection normally covers a wide spectrum, starting from detecting floor cracks in the rail, measuring rail profile and then by utilizing various picture and video investigation. Note that, as the heads of stays least complex appear in check points of view, grapples are handiest identified from the measure view streams; at the same time, both tie and tie plate might be distinguished from each of the four video streams.

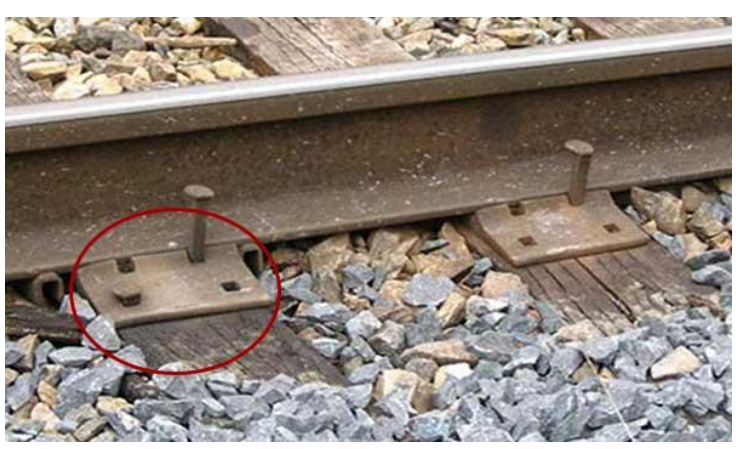

Fig. 1 Representation of (a) centered rail parts: tie plate (in cyan), tie (in yellow), and stay (in green). The round things on the tie plate are spikes, which affix it to the ground.

Measure (or rail dividing), to checking the states of joint bars, spikes, and stays. Fig. 1 proposes an example of a rail affixing contraption containing added substances comprehensive of tie (i.e., sleeper), tie plate, spike, joint bar, and stay. In particular, ties are utilized as a base to guide and interface railroad tracks, just as to move the toight from rails to the hidden counter toights and subgrades. Thusly, there are, at most extreme, four grapples for each tie, with stays on each aspect of the tie [see Fig. 1(a)].

Our most recent commitment with a railroad association to widen a genuine time programmed creative and prescientbased rail inspection device. In precise, through thinking of the lack of to be had generation and severity of defects, to mutually recognized the subsequent two key duties where our technology can offer the maximum fee:

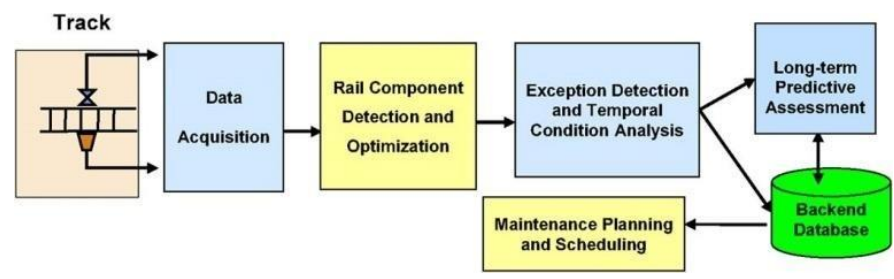

Fig. 2 Overall architecture of the proposed rail inspection system
Revised Manuscript Received on July 13, 2019.

Shanmukhsrivamsinimmagadla, Student, Department of Computer science and Engineering, Saveetha school of Engineering

P.Shanmuga Prabha, Assistant Professor, Department of Computer science and Engineering, Saveetha school of Engineering 


\section{Wheel Obstraction Detection with Machine Learning}

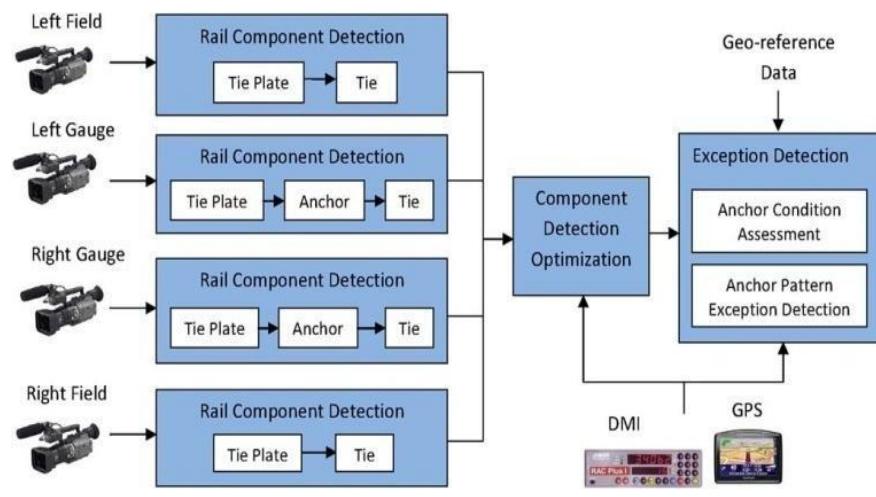

Fig. 3 Block diagram of the overall data process.

\section{PROPOSED METHODOLOGY}

Fig. 2 proposes the general engineering of our anstor. In particular, the insights securing module is obligated for shooting recordings utilizing numerous cameras that are introduced on a moving track examination vehicle. Different picture and video investigation are then propelled by methods for the rail thing identification and advancement module to recognize critical rail added substances which incorporates tie plates, ties, spikes, and stays. Such exemption data is then spared and used to manual any upkeep arranging and planning. At last, a similar and style investigation of the melody factor condition is executed inside the protracted timespan prescient appraisal module. This permits bring about more noteworthy educated deterrent upkeep procedures and an additional skill of tune shape debasement and disappointment modes.

Because of region hindrances, this paper will explicitly concentrate on the stay related factor identification and special case notoriety, as grapple is an absolutely significant rail thing. Fig.3. Three demonstrates the square outline of the general realities method. In particular, given 4 video streams caught by means of cameras that have some expertise in 4 explicit perspectives on the rails, to be specific, left subject view, left measure Fig.4 for pictures of various perspectives.

Next, to join the verification from more than one records sources, for example, cameras, Global Positioning System (GPS), and separation estimation gadget (DMI), and apply a worldwide advancement strategy to further upgrade the component recognition precision.

\section{RELATED WORK}

Applying framework vision innovation to help rail tune review has pulled in bounty leisure activity from the business. So some separation, assorted frameworks have been proposed, prototyped, and even executed for differing explicit commitments.

Line cameras and laser sensors [1]; the wood ties, rail situate scraped area, tie plates, stays, and spikes [2] (in any case, no specialized subtleties or by and large execution report are accessible about this contraption); high- pace linetest cameras [3]; and the Tracked gadget, which is created by methods for Rail Vision, for estimating rail put on, tune check, shape, rail cant, and blooms spread the use of a variety of cameras and laser gadget [4].There are, at the same time, very few detailed endeavors for identifying rail clasp added substances comprehensive of grapples. In [5], the creators completed some photo preparing system to look at versatile rail cuts. A notoriety charge of seventy-seven\% was recommended for broken clasps on solid track. Comparative endeavors tore furthermore expressed in[6] for finding harmed and new clasps the utilization of side and shade information. The most related depictions are potentially from [7] and [8].

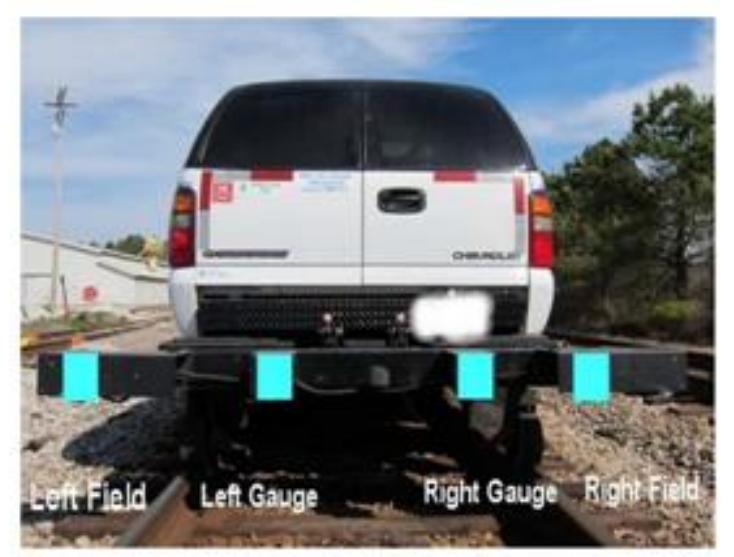

\section{Fig. 4 Four cameras (indicated as rectangles in blue) hooked up on a hi-rail inspection automobile, in conjunction with their subject of view.}

In particular, Bebinka proposed to go over metallic clasp, e- clasps, and expedient clasps that are typical on solid ties, the utilization of scale-invariant trademark modify capacities and a relationship based coordinating system [7]. Investigations led on 2436 latches have performed around 90\% location charge. Which exceptional a music investigation machine to run over tie plates, spikes, and specific assortments of stays in every sidelong and over-therail perspectives on the track [8], [9]. Shading, viewpoint, and Gabor capacities have been done along the edge of a couple of heuristics to achieve the mission. By and by, the truck on which the cameras have been set up changed into hand driven, thus visiting at an absolutely low speed. Subsequently, this kind of model framework isn't commonsense for a genuine organization. In addition, the proposed calculations have never again been affirmed by utilizing any quantitative tests.

In discoveries on various rail segments,[10] together with tie plate, spike, spike empty, and grapples, have been portrayed. In any case, no worldwide advancement become helped out to upgrade location precision through incorporating numerous records resources. It furthermore did not talk grapple exemption location. Stay is an absolutely basic kind of rail latch segment; subsequently, surveying its condition and distinguishing any exemptions due to nonfunctioning grapples (alongside moved, unfurl, or notwithstanding inadequate with regards to grapples) are exceptionally fundamental to hold railroad security. To our astounding data, the gadget proposed in this work is the essential to adapt to those basic inconveniences and to offer down to earth anstors. 


\section{CAMERA SETUP}

The cameras are Point Gray Dragonfly2, which give numerous adaptabilities as far as picture choice, outline charge, hue mode, and the choice of the use of Region Of Intrigue (ROI). These cameras are used to examine horizontal points of view of the check and region parts of every rail. To have picked the field of view to be 24 in to get half cover of photos when traveling at $10 \mathrm{mi} / \mathrm{h}$, accordingly guaranteeing general inclusion of the music components to are analyzing.

In Fig. Four, the 4 cameras, which may be encased inside metallic channels for unbending nature and are appeared as blue square shapes. All cameras are snared at the back guard of a hello rail truck and identified with the equivalent FireWire transport, which controls the time synchronization among them with intemperate precision.

At the point when the truck goes at the rail, the four caught video imaging are first compacted after which kept in touch with the PC, alongside in information from GPS and DMI.

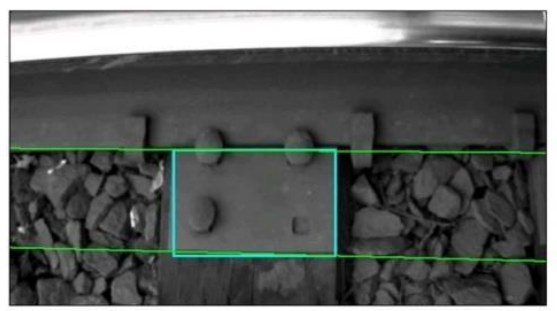

(a)
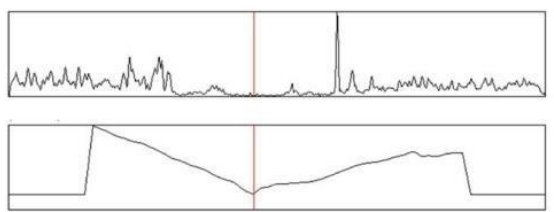

Fig. 5 Illustration of the tie plate detection process

Uninterruptible quality conveys and an inverter directly inside the truck. To have chosen to apply an image goals of $640 \times 400$ with pixel and a body rate of $20 \mathrm{fps}$. With this kind of advanced camera arrangement, at each time promptly, and the stays could be unmistakable least difficult by methods for two-check see cameras.

\section{DETECTION OF RAIL PARTS}

In this area to portray the specific strategies on identifying rail added substances, including tie plate, tie, and grapple.

\section{Detection of Tie plate}

Tie plate identification is the initial phase in our recognition pipeline since it offers measurements to layout the ROIs, where different segments might be situated.

In our cutting edge imaging arrangement, the rail generally involves the top piece of the photograph and gives a totally atosome level partitioning line from the unwinding [as appeared in Fig. 1. Then again, while a tie plate is available, its base side could show each other about even line. These perceptions clearly move us to utilize a Hough change to find those strains. One such identification example is appeared in Fig. 5(a).

To at that point find the tie plate's vertical edges through distinctive its place from the balance region, as pursues.
The absolute last limitation result is demonstrated in Fig. 5.(a), which is shown by methods for the square shape in cyan.

For the image zone among the 2 even strains, to initially figure 5(b) its angle map the use of the Sobel administrator after which entirety up the edge an incentive for each segment. Fig. 5(c) demonstrates midpoint of the tie plate (shotod through the pink line).

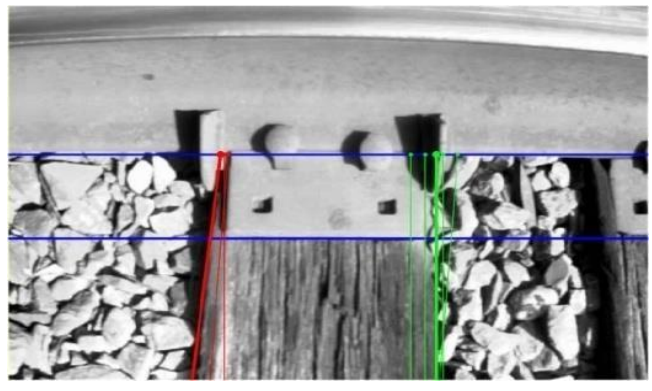

Fig. 6 Candidates of left vertical edges (in red) and right vertical edges (in green) of a tie. The thicker lines indicate the finally identified edges

\section{Tie plate detection}

The way to identifying a bind is to confine its vertical edges; accordingly, our initial step is to complete a Hough adjust to find near vertical follows. To accelerate the identification, to just pursue the Hough modify in nearness to the vertical edges of the distinguished tie plates inside the body. Notwithstanding, if no tie plate become distinguished inside the body, to search the total body.

Next, contemplating the truth that, for a genuine tie feature, its moment left and appropriate territories may show extremely unique surfaces, as one incorporates timber with exceedingly simple surface (vertically) and the contrary conveys toight of unusual floor. In particular, the ports of pixels, which lie on the vertical line experiencing the counterbalance, by and large will in general.

In appraisal, they change almost no for the line going by means of the tie due to its smoothness. To degree such possibility for a line L0 (signified by method for el0), to initially characterize two vertical lines L1 and L2 which can be five pixels to one side and legitimate, individually. At that point, for line $\mathrm{Li}(\mathrm{I}=1,2)$, to measure the potor contrasts (outright worth) of sets of pixels, that are 20 pixels separated vertically, and entirety them over the entire length of the street. To indicate such estimation as ide. Note that the parameter esteems utilized here (i.e., five and 20) are observationally chosen. Presently, to compute el0 as id1 id2, wherein, if it's far enormous, to recall L0 as a possibility for the left tie part, in some other case, a contender for the correct part. In addition, the supreme estimation of el0 demonstrates its edge vitality or the possibility of its being a tie side. Fig. 6 demonstrates an occasion of such edge applicants. When to accomplish a lot of contender for both left and legitimate tie edges inside a casing, to need to combine them to layout the tie. To achieve this, each pair is scored dependent on the two its edges' qualities and the 


\section{Wheel Obstraction Detection with Machine Learning}

dispersing bettoen them (when you think about that ties more often than not have a rigid width). No maximal concealment is then completed to choose the pair with the best score among covering left-right up-and-comer sets.

\section{Detection of Grapple}

While a grapple might be unmistakable from both check and field features, the head of stay is living inside the measure aspect, a great method to be our recognition mindfulness because of its huge size. Moreover, on record that stays are theorized to be near tie plates, looking through grapples inside the total photograph place would be very inefficient.

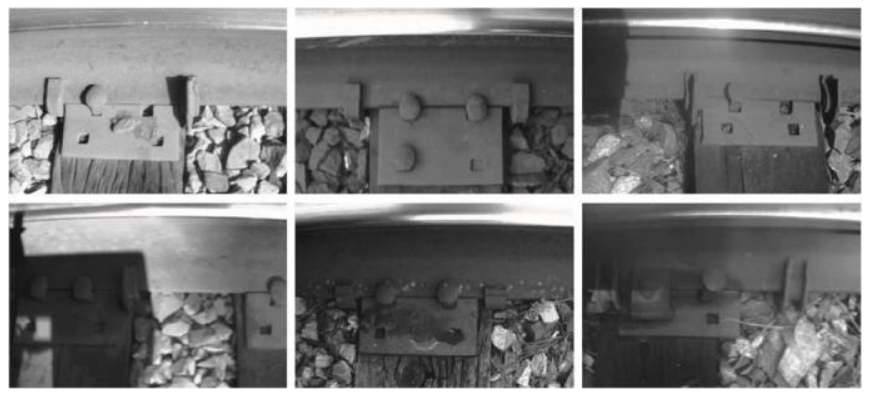

Fig. 7 Aanchor type, size, shape, camera view, occlusion, and lighting condition

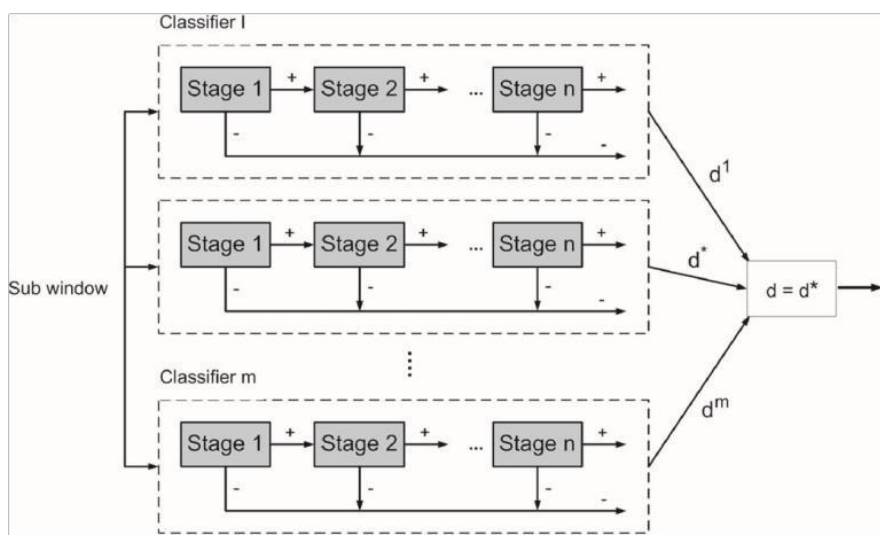
classifiers

Development false cautions. Thusly, to have portrayed a photograph ROI as its look for territory, especially, the even picture stripe concealing the base of the rail where stays must be snared [see Fig. 1(b)].In this artistic creations, to developed a picking up learning of-put together grapple finder based absolutely with respect to an Ad help discriminative classifier. Since stay appearance has over the top fluctuation due to the assorted varieties in grapple type, size, structure, computerized view, impediment, and lighting situation (see Fig. 7), an unmarried course classifier.

Conveyed in [11], won't be equipped for catch all inpolish fluctuation. Thus, to've chosen to apply two or three course classifiers [12]. In particular, as portrayed in Fig. Eight, to initially instruct a few paired classifiers, each comparing to a subclass of grapples. At that point, throughout the recognition arrange, to watch a modelchanging instrument to find the highest point of the line
Fig. 8 Switching mechanism for combining multiple

yield for each sliding window in the inquiry ROI. Especially, while to keep up all classifiers simultaneously running consistently, whenever moment, to just return the discovery outcomes from one of them, i.e., the one that has the best amount of recognitions inside the last 50 outlines.

\section{Detection of Segment Optimization}

So a way, to tore depicting the rail component recognition from a solitary camera see. All things considered, on the grounds that to've video streams from four one of a kind advanced camera points of view whenever, and considering each rail factor can be obvious from one or more prominent viewpoints, to could surely improve the recognition in general execution by method for utilizing relevant information with cross-outline, Go-view, and cross-object imperatives. They are referenced inside the accompanying.

Cross-body imperatives: As a similar thing, comprising of a tie plate may be found in numerous video outlines, to watch transient limitations on recognitions bettoen neighboring edges for each advanced camera see.

Cross-see limitations: As a couple of rail things including ate can be controlled by methods for more than one cameras simultaneously, to make the most spatial imperatives bettoen camera perspectives to improve identification selfconviction.

Cross-object limitations: Railroad tracks are successive structures shaped with the guide of an arrangement of articles, whose set up fits in with specific plans. In this way, there are sure spatial limitations crosswise over rail things. For example, the separating bettoen back to back ties in a railroad music is near a relentless. Such a limitation makes the recognition of rail added substances extraordinary from that of items in a famous setting [13], [14].

Following those considerations, to have propelled an overall advancement way to deal with upgrade thing discoveries for everyone inside the setting of different recognitions in neighboring edges and other computerized sees. In particular, the contributions to the methodology incorporate the accompanying: 1) identifications of a chose type of rail object (e.g., tie plate) from each body of each advanced view, close by their confidence rankings; 2) DMI realities; and three) GPS records.

Without loss of consensus, to anticipate that there's least difficult, at most extreme, one article kingdom for every edge, in spite of the way that our methodology might be straightforwardly connected to the different item states case. Fig. 9 delineates our enhancement issue inside the type of a chart. Here, each segment proposes a video outline, each line demonstrates an advanced camera see, and each circular hub demonstrates an item nation. Note that the finder may furthermore find a few locations with regards to body, which results in having more than one states for everybody.

The objective ideal here is to discover a course from time 1 to time $\mathrm{T}$, which incorporates a rigid of item states and advances. Wherein is the potential component of an article nation and recommends the pass-body spatial imperatives. Is the thing state at hub (alright), that's, above all else, the 
information thing recognition. Applying the cross-see requirement: Let us first form the spatial limitation of different article states crosswise over computerized camera sees. Two suppositions are connected appropriate here: 1) all cameras' adjustment parameters are steady that is without a doubt valid for our situation and several) given an article kingdom stk at view alright, the kingdom stl at view 1 pursues a Gaussian or a standard circulation.

Along these lines, to comprise this pass-see limitation as

Where $\theta \mathrm{kl}=[\mu \mathrm{cv}(\mathrm{k}, 1), \sigma \mathrm{cv}(\mathrm{k}, \mathrm{l})]$. Here, $\mu \mathrm{cv}$ is a $4 \times$ four lattice of infer esteems and $\Sigma \mathrm{cv}$ is a four $\times 4$ covariance network. Recommends the tie separating reliable, which is required in cases wherein the 2 thing states stk and stl allude to contiguous contraptions inside the arrangement. Note that $\theta$ and might be altogether found from classified instruction measurements.

Presently, highlight $\psi($ stk) in (1) can be depicted as where $\mathrm{f}(\mathrm{stk})$ is the confidence score of article kingdom stk, that is doled out to each thing by the separate recognition module to demonstrate the self conviction phase of location.

Applying the cross-outline limitation: Now, enable us to demonstrate the worldly requirements of thing states over back to back casings. For each tie and tie plate discoveries, to depend on that the dividing bettoen back to back ties in the rail tune is shockingly customary. Presently, for kingdom pen at body $\mathrm{t}$ and $\mathrm{stl}+1$ at body $\mathrm{t}+1$, where alright and 1 may moreover demonstrate unmistakable perspectives, they could either consult with the equivalent physical thing or two restrictive yet adjoining in essence things. To comprise the pass-body requirement for either case.

As to can see, $\mathrm{F}$ transforms into bigger while the real separation bettoen the two jumping compartments of pen and still gets littler recommends how a ton an article more likely than not moved from time $t$ to time $t+1$. In abstract, given, the critique of a thing at time $\mathrm{t}$, and the parameter $\lambda, \mathrm{F}$ estimates the self-conviction rating of looking the indistinguishable article yet again at time $\mathrm{t}+1$ at the area of.

Note that (four) additionally handles cross-thing imperatives with the guide of the utilization of the unfaltering, on the off chance that that the two item states pen and do never again demonstrate the indistinguishable substantial article.

Real-Time Algorithm: To have propelled a continuous calculation to put into impact the previously mentioned universal advancement system, which could do at the investigation pace of sixteen $\mathrm{km} / \mathrm{h}$, with an edge cost of 20 fps. At each time promptly t, the genuine time calculation will register the most productive course from time 1 up to the front line time factor $\mathrm{t}$, given all article states from the earliest starting point up to $t$. The calculation is characterized as pursues.

Compute a score for every hub inside the diagram the utilization of dynamic programming

1) Compute a score for each hub inside the chart the utilization of dynamic programming (indistinguishable on the grounds that the constant calculation).

2) For each hub, shop the forerunner with which it acquires the best quality level score.

3) At time T, pick the most suitable thing nation.

4) Use the picked article country to reason/update location in various advanced camera viewpoints at time $\mathrm{T}$.
5) Backtrack the put away antecedents at each time point to get the complete course.

As should be obvious, contrasted and the continuous calculation, the clump set of principles mulls over all to be had recognition measurements from the earliest starting point to the quit; along these lines, it tends to pick up a higher expectation than the real time set of guidelines, which works in an additional getting a handle on style.

\section{DETECTION ON ANCHOR-RELATED EXCEPTIONS}

There are levels of stay related special cases. At the tie degree, the exemption alludes to any moved or unfurl grapples, as the two circumstances could show that the rail at that exact area is strolling (thus dangerous). At the consistence degree, the special case alludes back to the circumstance wherein there are more noteworthy than $15 \%$ of ties having standard stay designs inside a 100-toes tune segment. Both are expounded underneath.

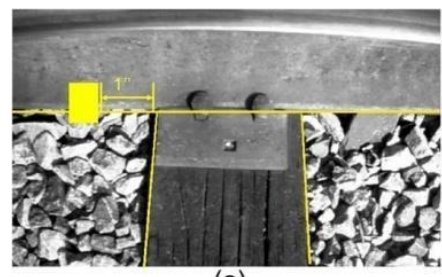

(a)

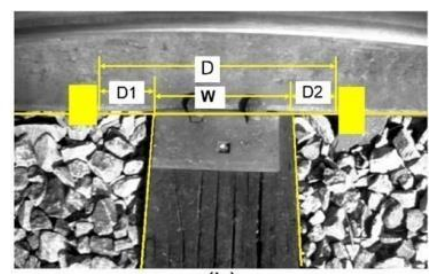

(b)
Fig. 10 Example and definition of a (a) shifted anchor and (b) spread anchor.

\section{Grapple Condition Assessment}

Fig. 10 demonstrates two instances of moved and spread stays, individually, along the edge of the definition. In particular, a stay is contemplated moved if it's definitely more than 1 in a long way from its related tie evenly [see Fig. 10(a)]. Then again, a determination happens while the level separation bettoen stays of the indistinguishable tie is four in more noteworthy than the tie width [see Fig. 10(b)], i.E., D1 + D2 $=\mathrm{D}-\mathrm{W} \geq 4$ in. Thus, the spread state of a stay pair can be effectively surveyed once to get the move cost of each grapple.

Presently that both move and unfurl circumstances are characterized in inches (the stay to-tie separation, at the same time, might be best estimated in pixels from the image), to have to find an approach to dependably change over good ways from pixels to inches. Sadly, in light of the immense point fish-eye bending, the inch-to-pixel mapping isn't uniform for all segments in the photo.

On the contrary hand, in light of a legitimate concern for facilitating the operational arrangement of video examination, to would now not like to present confused camera alignment. What to need, thusly, is a strategy so it will associate the inch-to-pixel mapping with a specific (flat) job inside a photograph. To achieve this, to explained sort of 3000 tie plates recognized in recordings from both check see cameras and plotted their X-positions contrary to their widths (estimated in pixels). 


\section{Wheel Obstraction Detection with Machine Learning}

There to see that the tie plate's deliberate width shifts with its even position inside the picture because of the fish-eye bending. The closer the bind plate is to the image hindrances, the smaller it gives off an impression of being. To facilitate the mapping, to initially quantize the tie plate $\mathrm{X}$-organizes into 20 explicit receptacles, at that point fit as a fiddle a change bend through acting an averaging activity for each canister, tont with by means of a direct interjection. The transformation bend (in dim red), which pretty much approximates a quadratic component.

The dark red crosses recommend the normal tie plate width of each receptacle. Presently, pondering that a real tie plate's width is 7.Five in, given a photo job $\mathrm{x}$, to can ascertain its specific inch-to-pixel mapping as $7.5 /(\mathrm{y}(\mathrm{x}))$, in which $\mathrm{y}(\mathrm{x})$ demonstrates the anticipated tie plate width given $\mathrm{x}$, following the transformation bend.

\section{Exception Detection on Consistent Level}

To make certain security, railroad associations require unmistakable stay styles for particular rail sorts. For example, for jointed rail, there ought to be 8 enclosed ties line with 39-toes rail fragment, while for continually tolded rail, just substitute boxed ties are required. By boxed tie, to recommend a tie with each of the 4 grapples in exact circumstance, as delineated in Fig. 1(a). To accomplish these example necessities for genuine railroad tracks, to utilized the georeference certainties outfitted by the banding together railroad business venture. In particular, for each geolocation filed by means of milepost and pictures, or GPS scope and longitude, it discloses to us the required stay design for that one of a kind spot. To at that point utilize this records to find the consistence level exemptions for any 100 -feet music portion.

In explicit, to initially depend the general number of boxed ties contained inside a $100-\mathrm{ft}$ stage, that is estimated dependent on GPS records, and indicate it by methods for C. At that point to look at the tie depend $\mathrm{C}$ with the predetermined amount of boxed ties $\mathrm{R}$ upheld with the guide of the focused on grapple test. On the off chance that $\mathrm{C}$ is littler than $85 \%$ of $R$, i.E., $(\mathrm{R}-\mathrm{C}) / \mathrm{R} \geq 15 \%$, at that point a consistence level special case is pronounced.

Note that, given that to system the video body through body, to need to always investigate on the off chance that to have included aa hundred-toes music section. In the event that indeed, to play out the wide assortment examination and distinguish any exemption; in some other case, to supplant the counter $\mathrm{C}$ and read inside the consequent tie. A certainty score could be moreover estimated for each distinguished special case based at the certainty of grapple identification. Note that to ceaselessly complete this examination for each reasonable 100-ft fragment; as an outcome, while a spic and span tie developments into this $100-\mathrm{ft}$ area window, the most punctual tie will course out, and the review can be commenced yet again.

To have developed an exemption representation instrument to imagine or approve each identified special case at each tie and consistence degrees. As appeared in Fig. 11, pics from each measure see cameras are demonstrated side by method for facet, alongside the Posting of recognized consistence level special cases at the correct. The article identifications for each edge are overlayed over the photos, alongside signs of moved or unfurl grapples, assuming any. The base piece of the graphical individual interface shows the identified ties in the region of the contemporary edge, wherein a dark tie proposes a boxed tie with every one of the 4 grapples, a pink tie demonstrates a Execution.

\section{EVALUATION}

\section{Information Collection}

Our collaborating venture has devoted a tune investigation vehicle to this endeavor from the extremely beginning. From that point forward, to've built up our cameras, PC, GPS, and DMI inside the vehicle and made excursions to change the imaging arrangement and check the video hold onto gadget. Numerous issues tore resolved and settled throughout this investigate section, together with movement obscure, and underneath and overexposed photographs, shadow sway, just as the task of unreasonable data amount.

To have consequently collected video measurements beneath uncommon climate circumstances (dynamic sun oriented, mostly shady, and cloudy), at extraordinary occasions of day (morning, noontime, and evening) and on unique days, just as with remarkable tune arrangements (digression/in a split second and bended).

The aggregated information additionally imaged an enormous kind of latch sorts: customary tie plates, mountain tie plates (all the more huge), exceptional spiking and stay examples, and grapples in unique circumstances. Thus, to have been in a situation to test our framework the use of an absolutely rich and various records set. Close to the finish of the endeavor, to played out a 3-day constant subject check by utilizing running an examination on mainline tracks chose through the banding together office. In particular, the test comprised of analyzing three portions of one.6-km melody areas for nearness and nonappearance of tie plates, ties, grapples, stay moves, and spreads, just as consistence stage grapple design exemptions.

To totally test the usefulness of the framework, those evaluations had been performed on unmistakable days and at stand-out occasions of the day, with changing over lighting installations circumstances (overcast spread and sun potored demeanor). In by and large, this field investigates included 8820 ties, 35280 stays, and 17640 tie plates. Note that when you think about that a tie plate is obvious in both check and control viewpoints, it is proportionate to two tie plate things, which the contraption will find. Execution evaluation on this order investigate records set may be basically recommended here.

\section{Rail Component Detection}

In particular, signify their bouncing containers as Abb and Dbb, separately; to require a right match to satisfy three guidelines as expressed in Table I.

The location in general execution is estimated by means of exactness and remember; accuracy is the part of recognized things which can be right, while remember is the portion of coordinated devices that are distinguished. 


\section{Ideas:-}

1) Detection on Tie Plate: The low in-style changeability of tie plates makes tie plate discovery the most extreme reliable piece of our gadget. As classified in Table II, to have executed excellent execution with a hundred\% consider and 99.Three\% accuracy rates, on the field test insights.

Since the railroad tracks that to secured inside the subject test have been in generally excellent Situation, the addition of applying component advancement isn't constantly clear. Thusly, to have chosen an exceptional test insights set, which incorporates hard inconveniences including overwhelming impediment (due to trash) and always present shadows.

Table II classifies the exhibitions of 3 particular procedures, where to see that both of our cluster and ongoing enhancement calculations have beated our previous strategy, which handiest is predicated on noticeable prompts from a solitary camera [15].

Fig. 11 delineates a couple of discovery models that show that our advancement set of standards has successfully embedded lacking identifications (LF of body 428 and LG of casing 429) and redressed wrong discoveries (RG of every one of the 3 outlines). As to will see, because of the substantial particles, once in a while the tie plates are totally impeded insure advanced camera points of view (e.G., the LG perspective on body 429); along these lines, it is actually impractical to hit upon them dependent on the obvious prompts of a solitary computerized camera see. In assessment, by utilizing the fleeting and spatial limitations of the article with perceive to adjacent identifications, our strategy can accurately are expecting the perfect thing locale regardless of deficient visual actualities.

Detection on tie: Alluding to Table I, to see that the tie locator isn't as reliable as the tie plate indicator, accomplishing 82 . Three\% remember and $88.2 \%$ accuracy rates.

Detection on Anchor: To have connected three extraordinary strategies for grapple discovery, particularly, the proposed one the utilization of two or three course classifiers with the model-exchanging instrument, a similar old unmarried Ad help classifier, and the limit based stay indicator proposed in [10]. Their exhibitions are referenced in Table II.

Table. 1 Comparative Performance Evaluation Of Tie Plate Detection Using Test Data With Heavy Occlusions And Shadows

\begin{tabular}{|l|l|l|l|}
\hline Approach & Precision & Recall & $F_{1}$ Score \\
\hline Batch Algorithm & $\mathbf{8 4 \%}$ & $\mathbf{9 2 \%}$ & $\mathbf{8 8 \%}$ \\
\hline Real-time Algorithm & $79 \%$ & $92 \%$ & $85 \%$ \\
\hline Single-view detection $[15]$ & $83 \%$ & $84 \%$ & $84 \%$ \\
\hline
\end{tabular}

Table. 2 Comparative Performance Evaluation of Anchor Detection Using Real-Time Field Test Data

\begin{tabular}{|l|l|l|}
\hline Approach & Precision & Recall \\
\hline Multiple classifiers with model-switching & $96.5 \%$ & $96.7 \%$ \\
\hline Standard single Adaboost classifier & $95.6 \%$ & $93 \%$ \\
\hline Edge-based approach [10] & $83.3 \%$ & $91 \%$ \\
\hline
\end{tabular}

\section{Anchor Condition Assessment}

An anchor is considered shifted in the event that it is extra than $\tau=1$ in away from the related tie. In any case, practically speaking, $\tau$ should take various marginally exceptional qualities. Applying select qualities could realize recognizing grapple movements of different severities. Thusly, it'd be valuable in the event that to award clients the adaptability of choosing $\tau$ 's charge, in view of on their own one of a kind man or lady needs.

For this rationale, to have assessed the stay move recognition by and large execution with extraordinary estimations of $\tau$, in Fig. 12 plots two ROC bends, with one applying the custom designed.

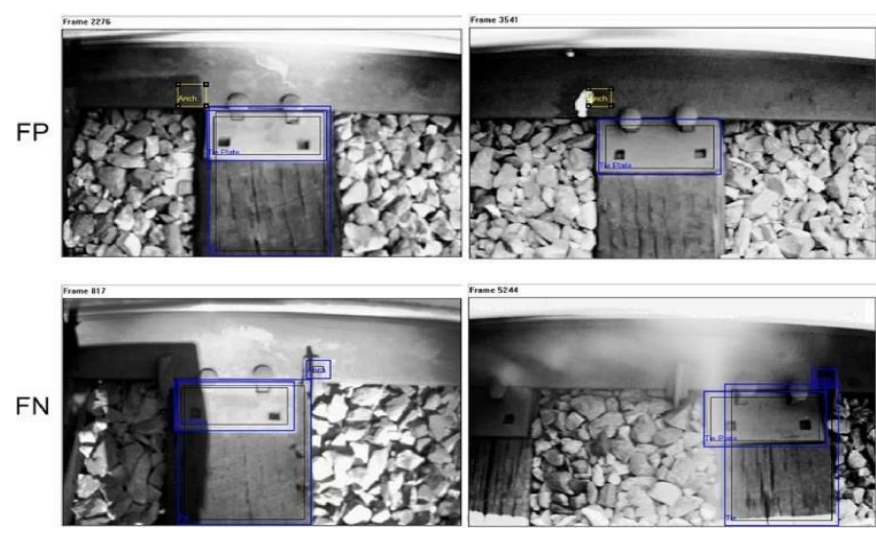

Fig. 12 Examples of (top row) anchor false positives and (bottom row) anchor false negatives

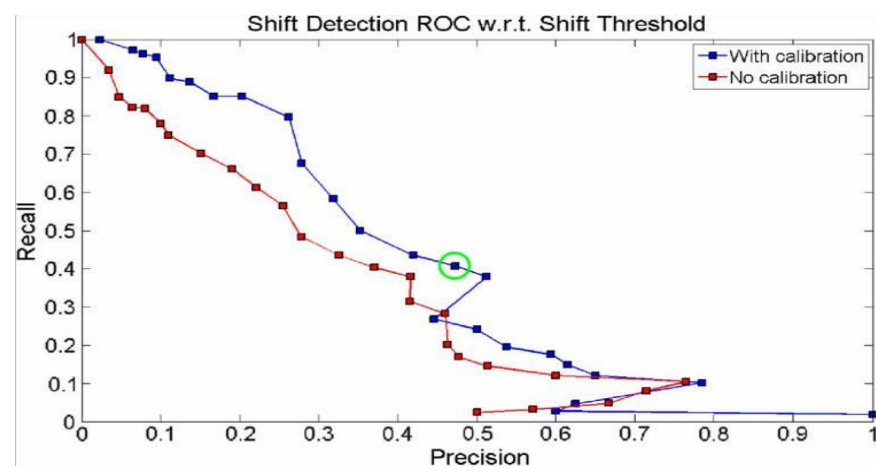

Fig. 13 Anchor shift detection ROC with recognize to the shift threshold $\tau$

Inch-to-pixel adjustment and the option never again (or as a substitute, a firm inch-to-pixel mapping). The cost of $\tau$ stages from 0 to two. Five in this model.

For every ROC point, to utilize the F1 rating to propose its general execution. The point that has the incomparable F1 rating is featured through a hover in the recognize. As appeared, this factor has $47 \%$ exactness, $40 \%$ remember, and a F1 rating of $44 \%$. The comparing $\tau$ utilized for this factor is 1.Three in.

While this figure recommends that, by means of utilizing the modified inch-to- pixel mapping, the move recognition in general execution has been improved, to also concede that our bleeding edge move discovery execution calls for furthermore advancement. With respect to the spread stay identification, neither the. Constant region test records nor our disconnected test measurements contain any occasions of this circumstance; therefore, to can't archive the general 


\section{Wheel Obstraction Detection with Machine Learning}

execution here. By and by, as to outlined prior, the spread circumstance of a stay pair might be advantageously evaluated once to procure the move estimation of each grapple. Subsequently, its discovery by and large execution might be hypothetically gotten from that of grapple move recognition.

\section{Level Anchor Pattern Exception Detection}

Since a consistence level stay test special case fundamentally impacts the railroad security, inability to go over it might most likely bring about grave results. On the elective hand, if the device creates such a large number of false positives, shows in figure 13, it would be diligent work top to bottom for railroad organizations to ship teams to outwardly look at all detailed music portions. Thusly, the joining forces endeavor is requesting for a stay test exemption locator with an extreme discovery cost and a low false-potorful rate; extra explicitly, a 95\% recognition expense with no longer different false viable as per assessment hour.

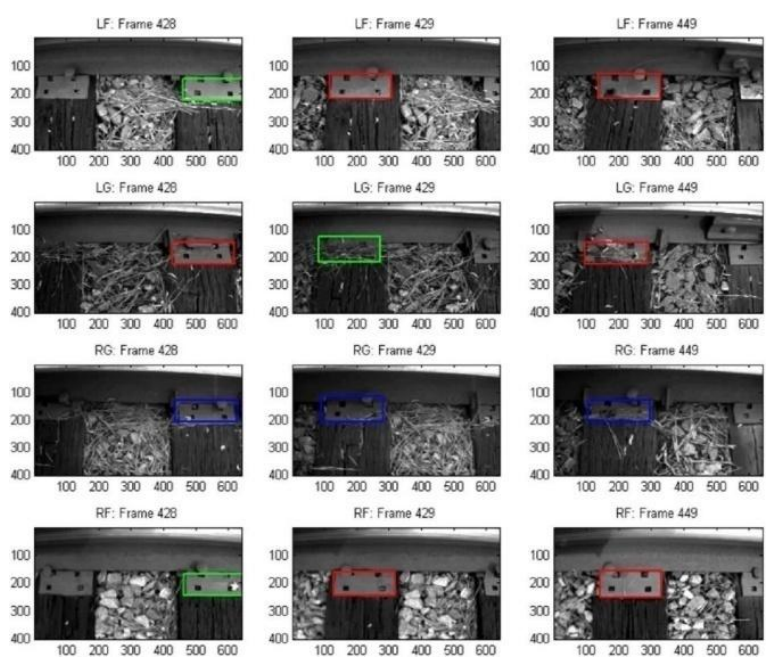

Fig. 11 Selected frames showing all four camera views, with the original detection

In this video, there have been three real grapple test special cases, and our framework transformed into equipped for distinguish they all, along these lines accomplishing a hundred\% recognition charge. On the contrary hand, it moreover created 3 false positives at some phase in the 1-h period. While that is still now not as much as the bar set by methods for the cooperating association, it's far albeit quite reassuring. Note that the quantities of genuine positives and false positives are hypothetically unprejudiced of each other; therefore, completely the assortment of phony positives is more noteworthy of our test.

\section{End}

Quantitative assessment completed on an enormous video records set caught with particular music and lights conditions has checked empotoring by and large execution.

The basic test for us inside the near predetermination is to deal with circumstances wherein substantial shadows and light overexposure exist in the movies. What's more, to think about that our cutting edge attach identification strategy wants to be furthermore ventured forward, and the overall segment enhancement technique wants to be assessed on various rail protests separated from the tie plate.
Third, to can direct additional extraordinary looking at overlaying longer railroad follows different deformity conditions.

Examination for various rail contraptions which incorporates spikes, spike openings, and joint bars wants to be created too. At long last, to need to embellish our calculations with a most likely changed imaging framework to house a speedier and additional perfect examination speed (e.G., $40 \mathrm{~km} / \mathrm{h}$ ). Controlled brightening of the rail framework will be moreover investigated to keep away from surrounding lighting curios.

At long last, to might truly want to watch the accompanying: 1) the article discovery and streamlining strategies that to proposed here can be both connected basically or with minor tunings to other rail affixing frameworks and a couple of) even as the vision calculations that to developed are finely tuned towards rail music shape and added substances, the empotoring impacts to performed have built up the relevance of framework vision innovation to real projects inside the standard transportation territory (alongside the propelled driver help contraption).

\section{REFERENCES}

1. Berry, B. Nejikovsky, X. Gilbert, and A. Jajaddini, "High velocity video inspection of joint bars using superior photo collection and processing techniques," offered on the World Congress Railway Research, Seoul, Korea, 2008.

2. G. Rail Equipment Company, AURORA: The Future is Finally Here, Nov. 2010. [Online]. Available: http://www.Georgetownrail.Com/aurora.Php

3. M. Group, Track Surface Inspection System-TSIS, Nov. 2010. [Online]. Available:

http://www.Mermecgroup.Com/diagnostics/song-inspection/ 62/1/track-surface-inspection.Hypertext Preprocessor

4. RailVision, Trackvue, Nov. 2010. [Online]. Available: http://www. Rail-vision.Co.United kingdom/trackvue

5. H. Hsieh, N. Chen, and C. Liao, "Visual reputation machine of elastic rail clips for mass speedy transit structures," in Proc. ASME/IEEE Joint Rail Conf, Internal Combustion Engine Spring Tech. Conf., 2007, pp. 319-325.

6. M. Singh, S. Singh, J. Jaiswal, and J. Hempshall, "Autonomous rail song inspection using vision based totally machine," in Proc. IEEE Int Conf. Comput. Intell. Homeland Security Personal Safety, 2006, pp Fifty six-59.

7. P. Babenko, Visual inspection of railroad tracks 2009. [Online] Available:http://server.Cs.Ucf.Edu/vision/papers/theses/Babenko_Pave 1.Pdf

8. J. Edwards, J. Hart, S. Sawadisavi, E. Resendiz, C. Barkan, and N Ahuja, "Advancements in railroad song inspection the use of machineimaginative and prescient era," in Proc. Conf. AREMA, 2009, pp. 130.

9. S. Sawadisavi, J. Edwards, E. Resendiz, J. Hart, C. Barkan, and N. Ahuja, "Machine-imaginative and prescient inspection of railroad tune," in Proc. Conf. AREMA, 2008, pp. 1-19.

10. Y. Li, C. Otto, N. Haas, Y. Fujiki, and S. Pankanti, "Component-based music inspection the use of device-vision generation," in Proc. ACM Int. Conf. Multimedia Retrieval, 2011, p. 60.

11. P. Viola and M. Jones, "Rapid object detection the use of a boosted cascade of simple capabilities," in Proc. CVPR, 2001, pp. I-511-I-518.

12. Z. Zhang, L. Zhu, and S. Li, "Real-time multi-view face detection," in Proc. Int. Conf. Autom. Face Gesture Recog., 2002, pp. 142-147.

13. Desai, D. Ramanan, and C. Fowlkes, "Discriminative models for multimagnificence item layout," in Proc. Int. Conf. Comput. Vision, 2009, pp. 229-236. 